\title{
An Electromagnet based Tablet Dispensing Device
}

\author{
Jamuna. C \\ Assistant Professor, Department of Biomedical \\ Engineering, Sri Shakthi Institute of Engineering and \\ Technology, Coimbatore, India.
}

\author{
Anjali Ravindran \\ Biomedical Engineer, \\ Ganga Hospital, Coimbatore, India
}

\section{PROPOSED SYSTEM}

Abstract- The home based health care devices provides an opportunity for the elderly people to remain safe and comfortable. Nowadays, in this busy schedule, people often forget to take medicines, which may lead to many fatal conditions. So keeping this in mind and to provide easy access to the aged people, this device has been designed. The dispensing device is mainly controlled by PIC microcontroller, the popping of the slot is accomplished using electromagnets. The medicines are popped out, as the user presses the switch. The software used in programming the PIC 16F877A microcontroller is C language in the MPLAB development environment using the HI-TECH C compiler.

Keywords-Dispensing device, Microcontroller, Electromagnets.

\section{INTRODUCTION}

The intake of medicines at the right time is a must for the elderly people. It is the responsibility of the family members to take care of them. But in the current scenario, people don't have time to take care of others. In order to provide a simple device, which will take care of the medications for the aged people, this work has been carried out. The main aim of the Tablet Dispensing Device is to reduce the medication errors and the fatal effects of it. Once the device is loaded with medicine and the time schedule is set, it reminds the patient to take the right medicine on time. The main step in the treatment of any disease is not just prescribing the medication, but also the right administration of the drug. Once the alarm is activated, the pill container pops up the tablet. The proposed model makes it easy, especially for the elderly people who has one or more chronic condition or those who have to take multiple medications.

\section{EXISTING METHODS}

\section{A. Automatic medical dispenser}

The device works automatically, and provided with 31 slots and this is the medication for entire ward and requires nurse supervision. It has 31 slots, used in hospitals. So it is very large, not portable.

\section{B. $\quad$ Dispensing device with motors}

Another existing system consists of many motors for controlling the dispenser unit. So it involves a complex circuitry.
The proposed design consists of electromagnets which are used for the popping mechanism. The design is very simple, since it does not involve the use of motors in it. It has a voice recording unit, which facilitates the user to record any type of voice commands, which can be used as a reminder for taking the medicines. It is cost effective and portable.

\section{METHODOLOGY}

Tablet Dispensing Device works by using an electromagnet. The power supply to the kit is delivered using a battery. The kit works in such a way that as the battery provides the supply to the kit, the PIC microcontroller provides the supply to the driver, thereby making the electromagnetic solenoid valve to pop up each slot as per the timing provided.

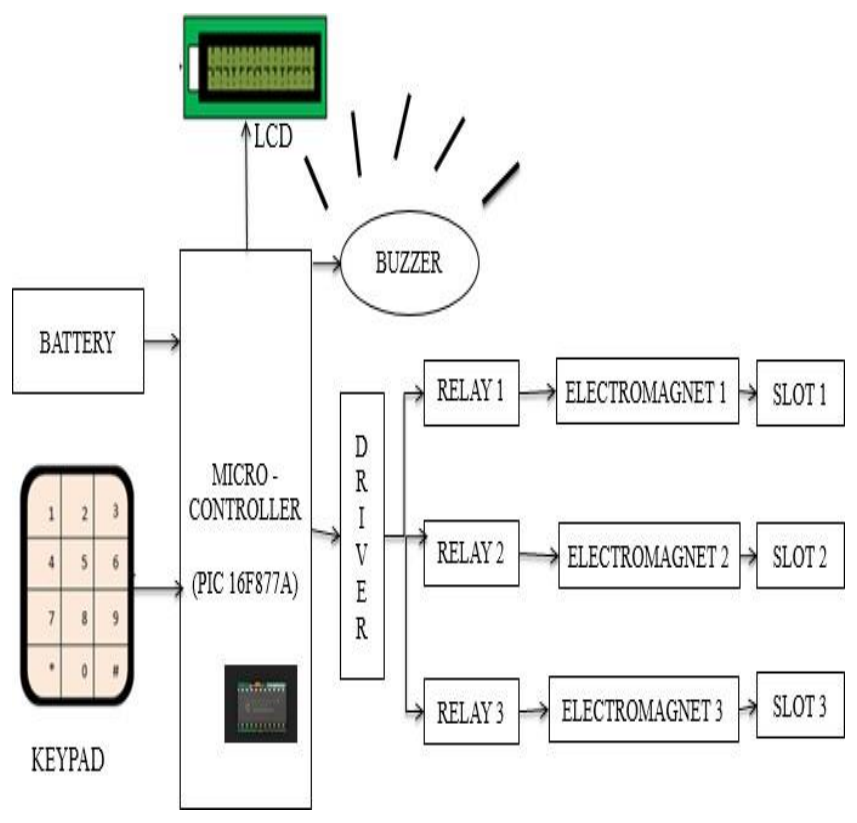

Fig.1. Block Diagram of the dispensing device 


\section{MECHANICAL HARDWARE}

\section{A. Battery}

This unit provides power supply for the working of all the units present in the block diagram right from the controller to the display. It is the prerequisite for the working of any electronic device. The controller and the related ICs work on a regulated supply of $5 \mathrm{~V}$ and other mechanical units work on a regulated supply of $12 \mathrm{~V}$.

\section{B. PIC microcontroller}

It is the heart on which the system thrives. The PIC 16F877A which has 32 lines as I/O ports is the one which we actually used in this work. Besides, it has an inbuilt analog to digital converter, timers and various other units and of course make use of easy embedded programming. This controller controls all the peripheral units connected to it including the electromagnet, alarm, voice recorder, switches.

\section{Drivers and relays}

Drivers are used to amplify the low voltage levels from the controller to drive the electromagnets. Relays are used to operate these electromagnets at desired time instants. They are used for driving three electromagnets. ULN 2003 IC is used as the driver and $12 \mathrm{~V}$ relays are used.

\section{Voice recorder \& buzzer}

The IC used in this unit is ISD 2500 which has many memory modules to store the voice commands to be generated at different intervals to aid the patients. The record and play mode aids the user to choose the desired settings and voice commands. The $5 \mathrm{~V}$ buzzer is used. Buzzer provides a continuous alarm until the user takes the medicine.

\section{E. Keypad}

In this design, two push buttons for entering the time and setting the time were used. One toggle switch is used as the power on switch. The push button at the top of the box is used for the pop up of tablets when pressed. The telephone keypad is used.

\section{F. $L C D$}

$16 * 2$ alpha numeric display is used as the LCD. The LCD is used in order to display the real timings, date, etc. It works in such a way that, when voltage is supplied the liquid crystal molecules get aligned and the light rays pass through the LCD and the desired characters are activated. Proper supply must be provided to avoid the flickering.

\section{G. Electromagnet}

A type of an electromagnet is known as a solenoid. It is often referred to as a solenoid valve. A solenoid valve has two main parts: the solenoid and the valve. The solenoid converts electrical energy into mechanical energy. The valve opens or closes due to this mechanism. A direct acting valve has only a small flow circuit. The electromagnet is mainly used to pop up the slot mechanically.

\section{OPERATING PROCEDURE}

The device is switched ON; it could be run on mains and also on battery. As the device is powered, LCD displays the real time and date. So after filling the slots with the pills, we have to enter the time using the keypad. Time can be set using the set time switch to which the alarm must remind. Once the set time is reached, the voice recorder gets activated followed by the buzzer that goes on until the person empties the slot. The device is designed for a day and the pills for an entire day can be placed in the three slots for morning, afternoon and night.

\section{RESULTS AND DISCUSSION}

The Tablet dispensing device is embedded in an acrylic box, so it is corrosion free, light weight and hence it is portable. The microcontroller, keypad matrix, LCD, battery and buzzer were interfaced in a printed circuit board which is shown in Fig.2.

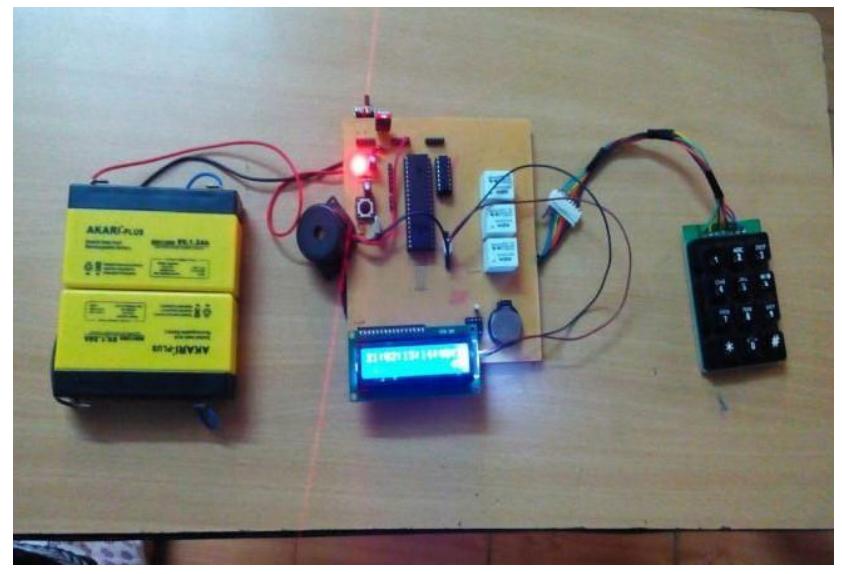

Fig.2. Interfacing of various modules

The entire setup including the pill boxes which are attached to the electromagnets, Voice recorder, battery, LCD, etc. was embedded in an acrylic box which is shown in Fig.3.

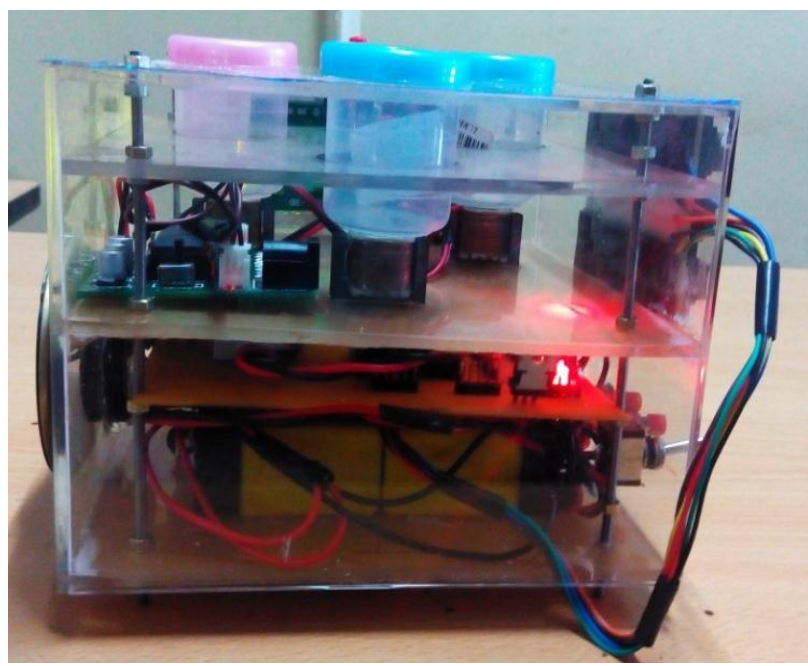

Fig.3. Dispensing device embedded in the acrylic box 
The time at which the medicine has to be popped up can be set in the device using the keypad attached to it and can be viewed in the LCD which is shown in Fig.4. Once the set time is reached, the device will give out an alarm sound along with the recorded voice command and then the tablet will be popped up from the corresponding slot.

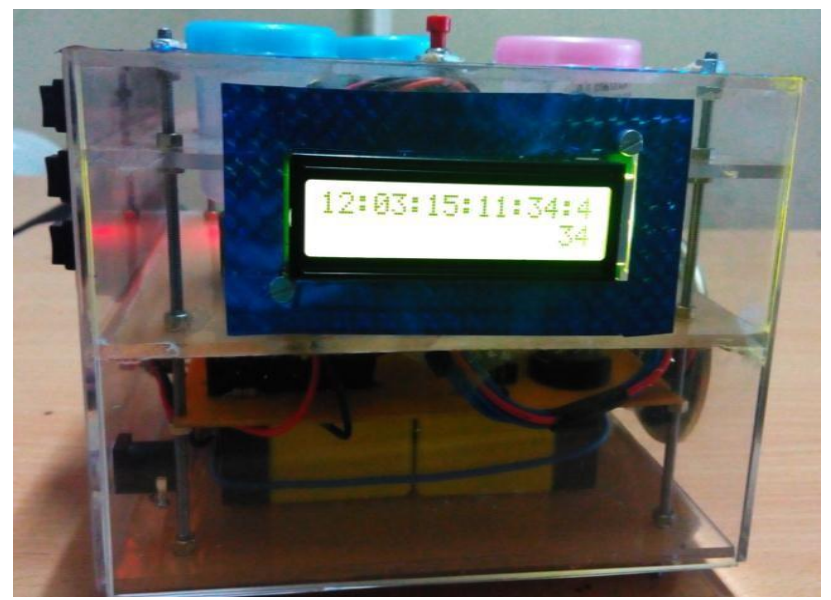

Fig.4. Device displaying the set time

\section{CONCLUSION}

Tablet Dispensing Device mainly works for pills and capsules of any desired size. The dispensing device can be programmed for a day and for any number of pills. It has the facility to give alarms three times a day. In fact, time can be fed in a sequence so that, on reaching a particular time the pill will be popped out. The proposed model aims at delivering the medicine at the right time and also it is cost effective, less weight and portable. The device also makes the users to be independent and also it reduces the risk of hospitalization due to wrong intake and missing schedules of medicine.

\section{FUTURE SCOPE}

In future, the device can be programmed to display the name of the medicine. It can further be modified by including the delivery of tonics.

\section{REFERENCES}

[1] Omair Shaikh, *et. al "Automatic Multipurpose Medicine Dispensing System", International Journal of Engineering and Technical Research (IJETR),Volume-3, pp 180-183, Issue-1, January 2015

[2] Sheeba abraham * et. al "Design of voice based pill ejector system," Biomedical and Pharmacology Journal ,Vol 7(1),pp 231-234, April 2014.

[3] Phyoe Sandarwin , * et. al " Pharmaceutical tablets counting and monitoring systems ", International Journal of science engineering \& technology research, Vol 3 , Issue 6, June 2014.

[4] Jagadeeshwar Vandakari, "Low cost robotic madical dispenser", International journal on computer science and engineering technology, Vol 02, No.08, pp 2648-2651, 2014
[5] AleksandrGeltsr, *et. al "Method and System For HighSpeed Tablet Counting and Dispensing", States, KirbyLester, Inc, 2014.

[6] S.Mukund ,* et. al " Design of automatic medication dispenser ," International journal of science and engineering and technology, Vol 3, pp 251-257, June 2012.

[7] Luciano Boquete, *et. al "Dynamically programmable electronic pill dispenser system", Journal of Medical Systems, 2010.

[8] Dr.A.S.C.S.S Astry ,*et. al "An automated microcontroller based liquid mixing system ,"International Journal on computer science and engineering , Vol 02 ,No.08, pp 2648-2651,2010.

[9] Coughlin, *et. al "Medicament Dispensing Cell", U. States. US, Scriptpro, LLC, Mission, KS, 2002.

[10] Keith@. Kudera, *et. al "Medicament Dispensing Cell with Dual Platens", U. States. US, Scriptpro LLC, Mission, Kans, 2000. 